\title{
Detection of "parent" molecules from the inner wind of AGB stars as tracers of non-equilibrium chemistry
}

\author{
L. Decin ${ }^{1,2, \star}$, I. Cherchneff ${ }^{3}$, S. Hony ${ }^{4,1}$, S. Dehaes ${ }^{1, \star \star}$, C. De Breuck ${ }^{5}$, and K. M. Menten ${ }^{6}$
}

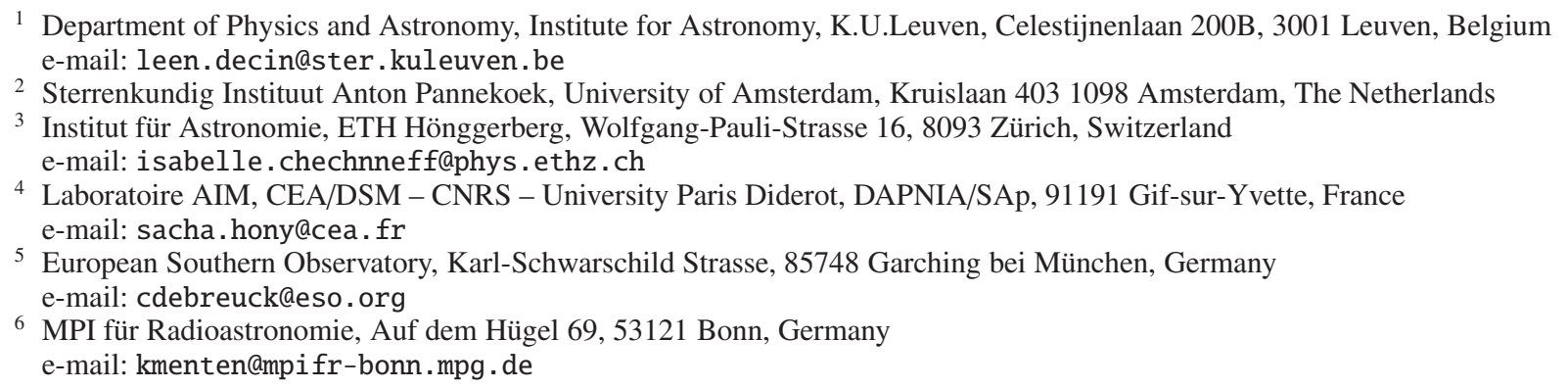

Received 22 October 2007 / Accepted 13 December 2007

\begin{abstract}
Context. Asymptotic Giant Branch (AGB) stars are typified by strong dust-driven, molecular outflows. For long, it was believed that the molecular content of the circumstellar envelope of AGB stars is primarily determined by the atmospheric C/O ratio. However, recent observations of molecules such as $\mathrm{HCN}, \mathrm{SiO}$, and $\mathrm{SO}$ reveal gas-phase abundances higher than predicted by thermodynamic equilibrium (TE) models. UV-photon initiated dissociation in the outer envelope or non-equilibrium formation by the effect of shocks in the inner envelope may be the origin of the anomolous abundances.

Aims. We aim to detect (i) a group of "parent" molecules (CO, SiO, HCN, CS), predicted by non-equilibrium studies to form with almost constant abundances independent of the $\mathrm{C} / \mathrm{O}$ ratio and the stellar evolutionary stage on the Asymptotic Giant Branch (AGB), and (ii) the few molecules, such as SiS and SO, that are sensitive to the O- or C-rich nature of the star.

Methods. Several low and high excitation rotational transitions of key molecules are observed at mm and sub-mm wavelengths with JCMT and APEX in four AGB stars: the oxygen-rich Mira WX Psc, the S star W Aql, and the two carbon stars V Cyg and II Lup. A critical density analysis is performed to determine the formation region of the high-excitation molecular lines.

Results. We detect the four "parent" molecules in all four objects, implying that, indeed, these chemical species form whatever the stage of evolution on the AGB. High-excitation lines of SiS are also detected in three stars with APEX, whereas SO is only detected in the oxygen-rich star WX Psc.

Conclusions. This is the first multi-molecular observational proof that periodically shocked layers above the photosphere of AGB stars show some chemical homogeneity, whatever the photospheric $\mathrm{C} / \mathrm{O}$ ratio and stage of evolution of the star.
\end{abstract}

Key words. astrochemistry - molecular processes - stars: AGB and post-AGB - stars: circumstellar matter - stars: mass-loss submillimeter

\section{Introduction}

Circumstellar envelopes of Asymptotic Giant Branch stars (AGBs) have long been known to be efficient sites of molecule formation. While the outer layers of such envelopes experience penetration of interstellar UV photons and cosmic rays resulting in a fast ion-molecule chemistry, the deepest layers are dominated by a non-equilibrium chemistry due to the passage of shocks generated by stellar pulsation. Dust forms in those inner gas layers, still bound to the star, and grains couple to the gas to accelerate it, thereby generating stellar wind and mass loss phenomena. The described processes greatly modify the abundances established by the equilibrium chemistry in the dense, hot photosphere (Tsuji 1973).

For a long time, the gas chemical composition was believed to be dominated entirely by the $\mathrm{C} / \mathrm{O}$ ratio of the photosphere. A

\footnotetext{
$\star$ Postdoctoral Fellow of the Fund for Scientific Research, Flanders.

$\star \star$ Scientific Researcher of the Fund for Scientific Research, Flanders.
}

$\mathrm{C} / \mathrm{O}$ ratio greater than one implied that all the oxygen was tied in $\mathrm{CO}$, leading to an oxygen-free chemistry, whereas a $\mathrm{C} / \mathrm{O}$ ratio of less than one meant that no carbon bearing molecules apart from $\mathrm{CO}$ could ever form in an oxygen-rich (O-rich) environment. This picture, based essentially on thermal equilibrium considerations applied to the gas, has been disproved by the detection of $\mathrm{SiO}$ at millimeter $(\mathrm{mm})$ wavelength in carbon-rich (C-rich) AGBs (e.g. Bujarrabal et al. 1994; Schöier et al. 2006b). As for O-rich AGBs, $\mathrm{CO}_{2}$ infrared (IR) transition lines were detected in various objects with the Short-Wavelength Spectrometer (SWS) onboard the Infrared Space Observatory (ISO) (e.g., Justtanont et al. 1996; Ryde et al. 1998).

Theoretical modeling describing the chemistry in the inner wind of the extreme carbon star IRC+10216 showed that the formation of $\mathrm{SiO}$ was due primarily to hydroxyl $\mathrm{OH}$ reaction with atomic silicon close to the photosphere as a result of shock activity and therefore non-equilibrium chemistry (Willacy \& Cherchneff 1998). Later on, Duari et al. (1999) showed that 
$\mathrm{CO}_{2}$ formation in the O-rich Mira IK Tau results from the reaction of $\mathrm{OH}$ radicals with $\mathrm{CO}$ in the shocked regions, implying again that non-equilibirum chemistry was crucial for the formation of C-bearing species in O-rich Miras. It was then recently proposed that the inner wind of AGBs shows a striking homogeneity in chemical composition, independent of their photospheric $\mathrm{C} / \mathrm{O}$ ratio and stage of stellar evolution (Cherchneff 2006). In particular, Cherchneff (2006) showed that when taking shock chemistry into account, molecules such as $\mathrm{SiO}, \mathrm{HCN}$ and $\mathrm{CS}$ are present in comparable amounts in the inner layers of $\mathrm{M}$, $\mathrm{S}$, and C AGBs, whereas specific molecules (e.g. SO and HS for O-rich Miras and $\mathrm{C}_{2} \mathrm{H}_{2}$ for carbon stars) are typical of O-rich or C-rich chemistries.

In this letter, we present observations carried out with the JCMT and the APEX telescope of four AGBs: one O-rich, WX Psc, one $\mathrm{S}$ star $(\equiv \mathrm{C} / \mathrm{O} \approx 1), \mathrm{W} A q l$, and two carbon stars, II Lup and V Cyg. We focus on the detection of (sub)mm transitions of $\mathrm{CO}, \mathrm{SiO}, \mathrm{HCN}, \mathrm{CS}, \mathrm{SiS}$ and $\mathrm{SO}$ in order to confirm or disprove the above hypothesis and to check for homogeneity in AGB winds.

\section{Observations and line profiles}

The observations were performed in October 2006 with the $15 \mathrm{~m}$ $\mathrm{JCMT}^{1}$ for V Cyg, WX Psc, and W Aql, and in the period from September to October 2006 with the APEX ${ }^{2} 12$ m telescope for II Lup, WX Psc and W Aql. Due to technical problems with the RxB3 JCMT receiver, only low frequency lines within the RxA3 receiver (211-276 GHz) were obtained. For the APEX observations, both the APEX-2A receiver $(279-381 \mathrm{GHz})$ and FLASH receiver (460-495 GHz and 780-887 GHz) were used. The observations were carried out using the position-switching mode. The JCMT data reduction was performed with the SPLAT devoted routines of STARLINK, the APEX-data with CLASS. A polynomial was fitted to an emision free region of the spectral baseline and subtracted. The velocity resolution for the JCMTdata is $0.0305 \mathrm{MHz}$, and for the APEX-data is $0.1221 \mathrm{MHz}$. For WX Psc, W Aql, and II Lup the data were rebinned to a resolution of $1 \mathrm{~km} \mathrm{~s}^{-1}$, for V Cyg to $0.75 \mathrm{~km} \mathrm{~s}^{-1}$ in order to have at least 40 independent resolution elements per line profile. The antenna temperature, $T_{\mathrm{A}}^{*}$, was converted to the main-beam temperature $\left(T_{\mathrm{mb}}=T_{\mathrm{A}}^{*} / \eta_{\mathrm{mb}}\right)$, using a main-beam efficiency $\eta_{\mathrm{mb}}$ of 0.69 for the JCMT RxA3 receiver, of 0.73 for the APEX-2A receiver, and of 0.60 and 0.43 for the $460-495 \mathrm{GHz}$ and $780-887 \mathrm{GHz}$ FLASH channels respectively (Güsten et al. 2006).

The observed molecular emission lines of four AGB stars in our sample are displayed in Figs. 1-4.

In all stars molecular emission lines of $\mathrm{CO}, \mathrm{SiO}, \mathrm{HCN}$, and $\mathrm{CS}$ are detected. This confirms the prediction of homogeneity by Cherchneff (2006) as these species are "parent" molecules that form in the inner layers of the CSE. This can be understood in terms of the chemistry of these four molecules being determined by shock propagation and not by the photospheric $\mathrm{C} / \mathrm{O}$ ratio and the stellar evolutionary stage.

1 The James Clerk Maxwell Telescope (JCMT) is operated by The Joint Astronomy Centre on behalf of the Science and Technology Facilities Council of the UK, The Netherlands Organisation for Scientific Research, and the National Research Council of Canada. Program ID is m06bn03 (34 h).

2 APEX, the Atacama Pathfinder Experiment, is a collaboration between the Max-Planck-Institut fur Radioastronomie, the European Southern Observatory, and the Onsala Space Observatory. Program ID is ESO.078.D-0534 (44 h).
SiS is also detected in all stars and with APEX we were able to detect the high-excitation $\operatorname{SiS}(19-18)$ line in the O-rich WX Psc, the C-rich II Lup and the S-rich W Aql. This is in good agreement with recent SiS OSO, JCMT, and APEX observations of Schöier et al. (2007) in a large sample of M and C stars, including WX Psc and V Cyg. This implies that SiS also forms close to the star, whatever the stage of stellar evolution.

Both the $\mathrm{SO}\left(6_{5}-5_{4}\right)$ and the high-excitation $\mathrm{SO}\left(10_{11}-10_{10}\right)$ line were detected in O-rich WX Psc. SO was neither found in the S-type W Aql, nor in the two carbon stars II Lup and V Cyg. SO appears to be typical for O-rich AGBs only, supporting the non-detection of SO in C-stars by Woods et al. (2003).

Both optically thin and optically thick lines occur (e.g., ${ }^{13} \mathrm{CO}(2-1)$ versus the ${ }^{12} \mathrm{CO}(2-1)$ line in W Aql, see e.g. Fig. 2). The line parameters, i.e., the main-beam brightness temperature at the line centre $\left(T_{\mathrm{mb}}\right)$, the line centre velocity $\left(v_{*}\right)$, and half the full line width $\left(v_{\mathrm{e}}\right)$, are obtained by fitting the "soft parabola" line profile function to the data (Olofsson et al. 1993)

$T(v)=T_{\mathrm{mb}}\left[1-\left(\frac{v-v_{*}}{v_{\mathrm{e}}}\right)^{2}\right]^{\beta / 2}$,

where $\beta$ describes the shape of the line. The velocity-integrated intensities are obtained by integrating the emission between $v_{*} \pm v_{\mathrm{e}}$ and are listed in Table 1 . A value of $\beta=2$ represents a parabolic line shape, expected for optically thick expanding spherical envelopes if the source is much smaller than the radio telescope beam size; $\beta<0$ results in a profile with horns at the extreme velocity, expected for optically thin lines when the source is resolved (Morris 1975). In our sample, several lines have a $\beta$-value distinctly larger than 2 , indicating that the line formation occurs partially in the inner region, where the stellar wind has not yet reached its full terminal velocity. This is notably the case for the $\mathrm{CS}, \mathrm{SiO}$, and $\mathrm{HCN}$ lines in our sample, corroborating their formation close to the star. The estimated terminal velocities for WX Psc, W Aql, II Lup, and V Cyg are $19.2 \mathrm{~km} \mathrm{~s}^{-1}, 18.8 \mathrm{~km} \mathrm{~s}^{-1}, 20.1 \mathrm{~km} \mathrm{~s}^{-1}$, and $12.4 \mathrm{~km} \mathrm{~s}^{-1}$, respectively. A maximum of $5 \%$ difference is found in the derived terminal velocity values for each target, being within the velocity binsize, indicating that all of the detected lines at least partly survive the dust formation and wind acceleration processes. An exception is $\mathrm{SiS}(19-18)$ in WX Psc $\left(16 \mathrm{~km} \mathrm{~s}^{-1}\right)$, but particularly the $\mathrm{SO}\left(10_{11}-10_{10}\right)$, with an estimated terminal velocity of only $10 \mathrm{~km} \mathrm{~s}^{-1}$, traces a much smaller geometrical region.

\section{Excitation analysis}

If many transitions of an individual molecule can be observed, it is possible to assess whether collisional or radiative excitation mechanisms can produce the observed line intensities. While it is well known that $\mathrm{CO}$ is formed in both $\mathrm{M}, \mathrm{C}$ and S-type stars and survives dust condensation, it is of interest to study the excitation requirements for the three other "parent" molecules $\mathrm{SiO}$, $\mathrm{HCN}$ and CS predicted by Cherchneff (2006) to be abundant in the inner winds of $\mathrm{M}, \mathrm{S}$ and $\mathrm{C}$ AGBs.

For all lines, except for those from the $\mathrm{CO}$ molecule, the emission distribution is expected to be much smaller than the FWHM beam size of the telescope used. To calculate column densities we need to correct for the different beam filling factors, $f=\theta_{\mathrm{S}}^{2} /\left(\theta_{\mathrm{S}}^{2}+\theta_{\mathrm{B}}^{2}\right)$, with $\theta_{\mathrm{B}}$ and $\theta_{\mathrm{S}}$ being the FWHM of the beam and the source respectively. To do this, we define a beamaveraged brightness temperature, $T_{\mathrm{b}}$, scaled by correcting our main-beam brightness temperatures for a fictitious 10" FWHM source, i.e., $T_{\mathrm{b}}=1 / f \times T_{\mathrm{mb}}$. 
L. Decin et al.: Non-equilibrium chemistry in AGB stellar winds
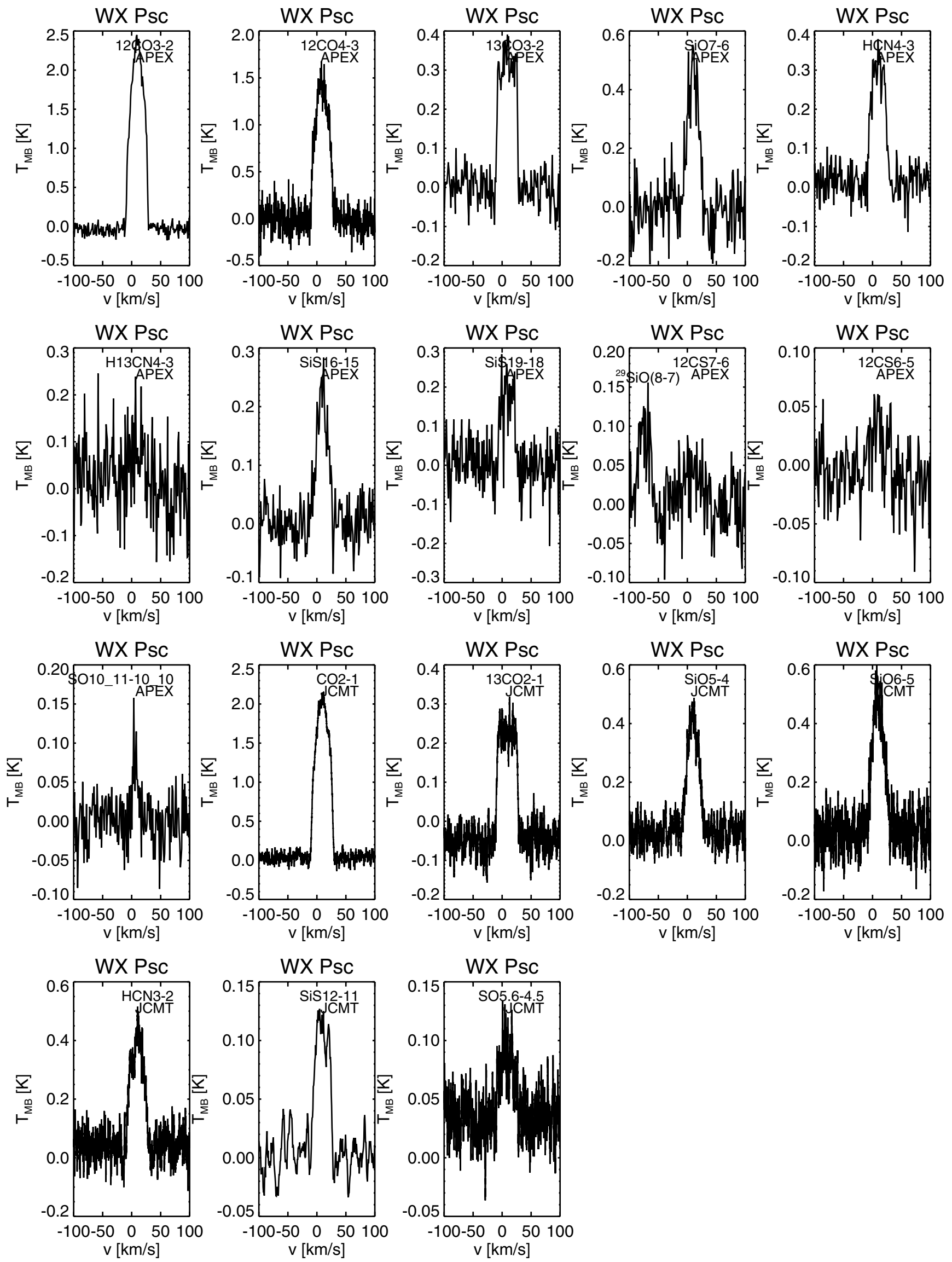

Fig. 1. Molecular emission detected with APEX and JCMT in the O-rich AGB WX Psc. 

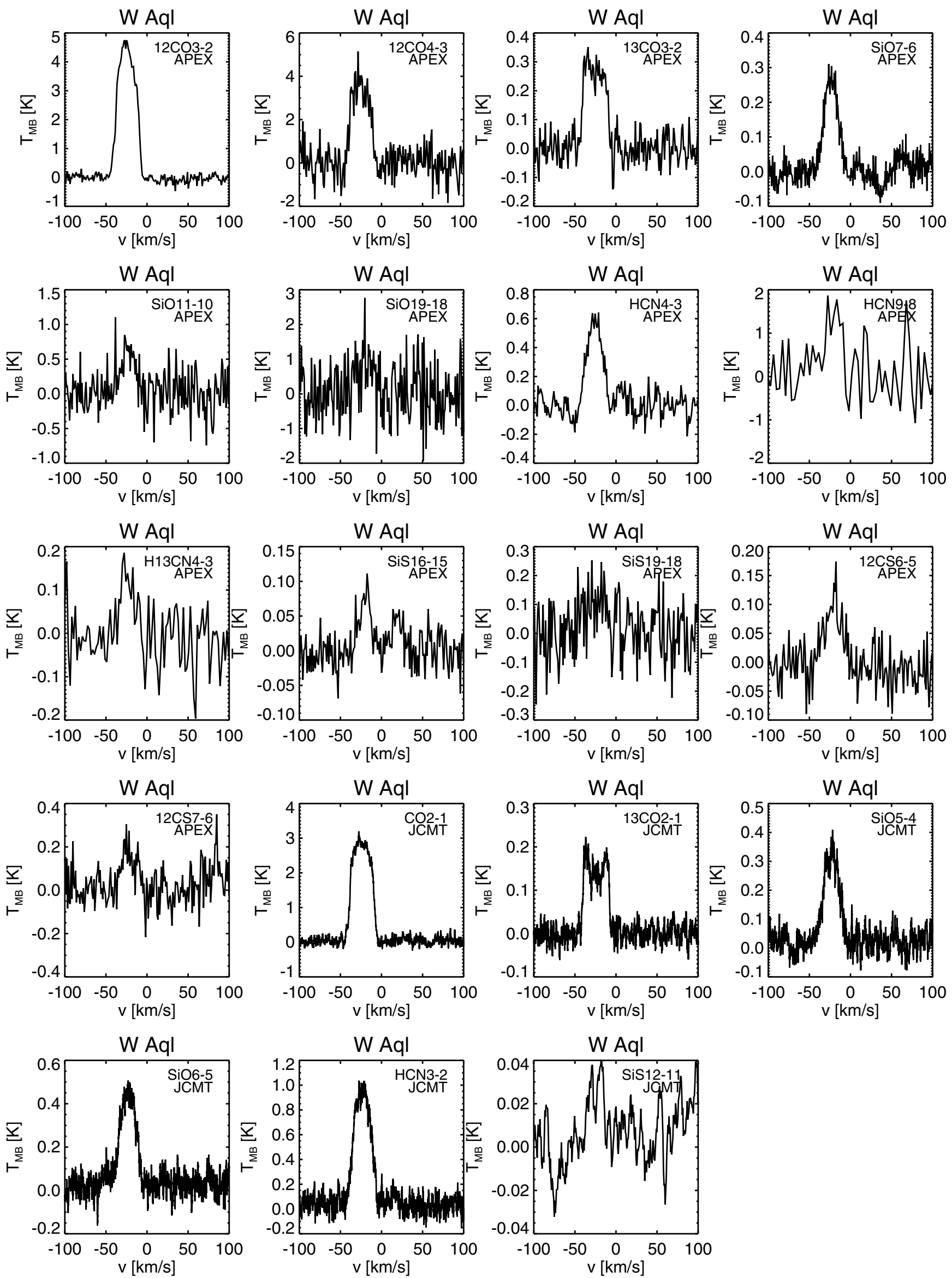

Fig. 2. Molecular emission detected with APEX and JCMT in the S-type AGB W Aql. Note that the HCN(9-8) line is rebinned to a resolution of $3.6 \mathrm{~km} \mathrm{~s}^{-1}$, other lines are rebinned to a resolution of $1.8 \mathrm{~km} \mathrm{~s}^{-1}$. 

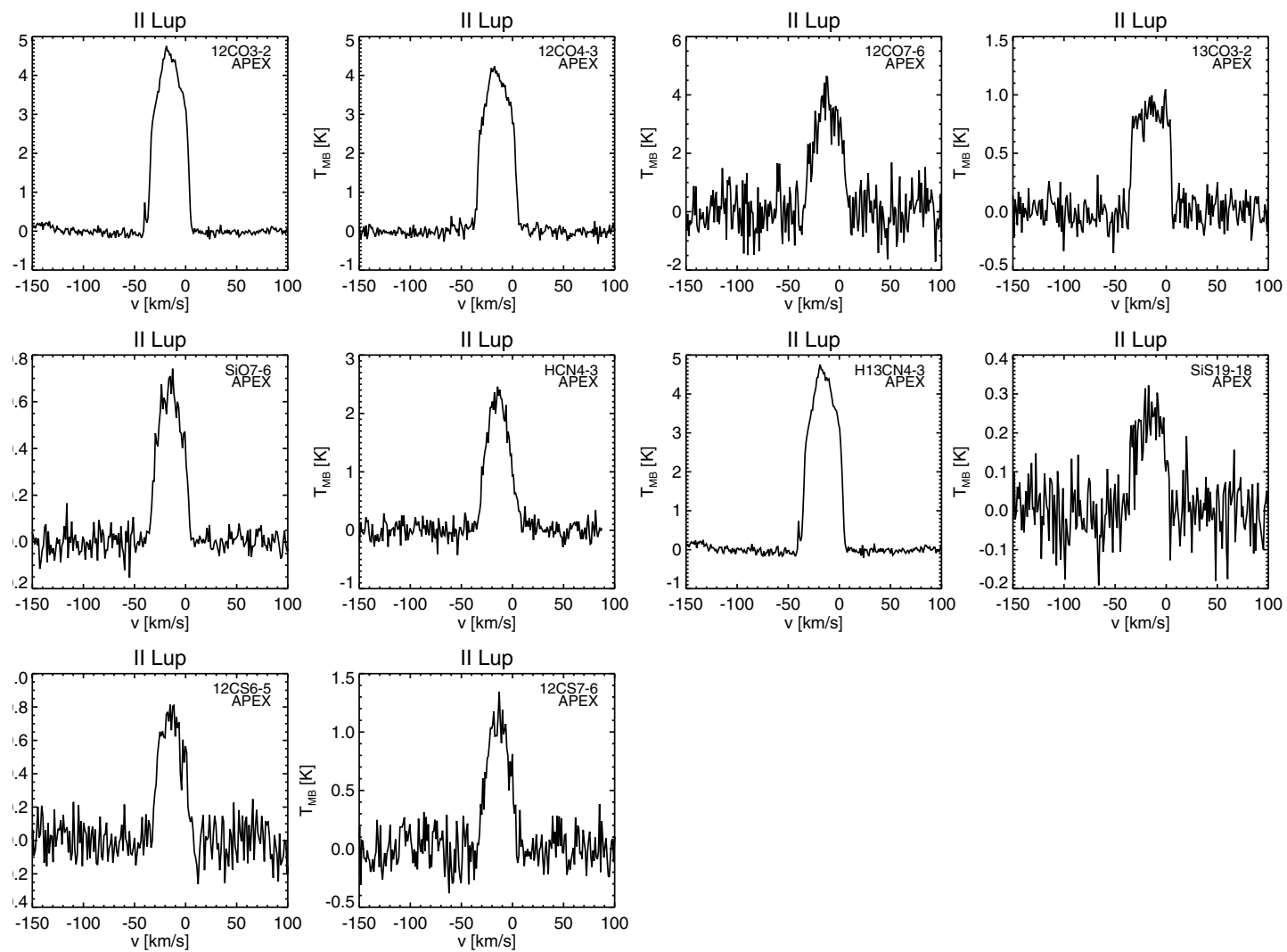

Fig. 3. Molecular emission detected with APEX in the C-rich AGB II Lup.

Table 1. Overview of the velocity-integrated intensities $\left(\int T_{\mathrm{mb}} \mathrm{d} v\right.$ in $\left.\mathrm{K} \mathrm{km} \mathrm{s}^{-1}\right)$ for the observed line transitions. The frequency is listed in GHz and the lower energy level in $\mathrm{cm}^{-1}$. The integrated intensity of detected lines with a low S/N-ratio is given between brackets. In case of a non-detection, an upper limit on the integrated intensity is computed as $3 \sigma \times$ expected linewidth, with $\sigma$ the noise on the data. A "-" indicates lines that were not observed. APEX-data are reported in plain front, JCMT-data are given in italics.

\begin{tabular}{|c|c|c|c|c|c|c|c|c|c|c|c|}
\hline Transition & ${ }^{12} \mathrm{CO}(2-1)$ & ${ }^{12} \mathrm{CO}(3-2)$ & ${ }^{12} \mathrm{CO}(4-3)$ & ${ }^{12} \mathrm{CO}(7-6)$ & ${ }^{13} \mathrm{CO}(2-1)$ & ${ }^{13} \mathrm{CO}(3-2)$ & ${ }^{12} \mathrm{CS}(5-4)$ & ${ }^{12} \mathrm{CS}(6-5)$ & ${ }^{12} \mathrm{CS}(7-6)$ & ${ }^{12} \mathrm{CS}(10-9)$ & ${ }^{12} \mathrm{CS}(17-16)$ \\
\hline frequency & 230.538 & 345.795 & 461.040 & 806.651 & 220.398 & 330.587 & 244.935 & 293.912 & 342.882 & 489.759 & 832.061 \\
\hline$E_{\text {low }}$ & 3.84 & 11.54 & 23.07 & 80.74 & 3.67 & 11.03 & 16.34 & 24.51 & 34.32 & 75.53 & 222.15 \\
\hline WX Psc & 58.0 & 64.3 & 40.2 & 22.4 & 9.9 & 11.3 & $<2.4$ & {$[0.8]$} & 1.3 & $<8.1$ & $<42.6$ \\
\hline W Aql & 80.6 & 118.4 & 95.9 & $<178$ & 4.6 & 8.1 & - & 2.4 & 4.6 & $<54.1$ & $<203$ \\
\hline II Lup & - & 145.0 & 130.0 & 99.7 & - & 32.5 & - & 20.4 & 35.2 & - & - \\
\hline V Cyg & 45.6 & - & - & - & 4.2 & - & 7.4 & - & - & - & - \\
\hline Transition & ${ }^{13} \mathrm{CS}(7-6)$ & ${ }^{13} \mathrm{CS}(10-9)$ & $\mathrm{H}^{12} \mathrm{CN}(3-2)$ & $\mathrm{H}^{12} \mathrm{CN}(4-3)$ & $\mathrm{H}^{12} \mathrm{CN}(9-8)$ & $\mathrm{H}^{13} \mathrm{CN}(4-3)$ & $\mathrm{SiO}(5-4)$ & $\mathrm{SiO}(6-5)$ & $\mathrm{SiO}(7-6)$ & $\mathrm{SiO}(11-10)$ & \\
\hline frequency & 323.684 & 462.334 & 265.886 & 354.505 & 797.433 & 345.340 & 217.105 & 260.518 & 303.926 & 477.504 & \\
\hline$E_{\text {low }}$ & 32.39 & 69.41 & 8.87 & 17.74 & 106.42 & 17.28 & 14.48 & 21.73 & 30.42 & 79.65 & \\
\hline WX Psc & $<67.4$ & $<12.7$ & 9.9 & 8.9 & $<117$ & {$[2.6]$} & 9.2 & 11.1 & 10.4 & $<15.0$ & \\
\hline W Aql & - & $<13.0$ & 21.7 & 12.2 & [34.1] & 3.1 & 7.1 & 8.9 & 5.2 & 13.8 & \\
\hline II Lup & - & - & - & 60.7 & - & 64.2 & - & - & 19.6 & - & \\
\hline V Cyg & - & - & 25.7 & - & - & - & 3.8 & 5.1 & - & - & \\
\hline Transition & $\mathrm{SiO}(19-18)$ & $\mathrm{SiS}(12-11)$ & $\mathrm{SiS}(14-13)$ & $\mathrm{SiS}(16-15)$ & SiS(19-18) & $\mathrm{SO}\left(1_{1}-1_{0}\right)$ & $\mathrm{SO}\left(6_{5}-5_{4}\right)$ & $\mathrm{SO}\left(7_{8}-6_{7}\right)$ & $\mathrm{SO}\left(8_{9}-8_{8}\right)$ & $\overline{\mathrm{SO}\left(10_{11}-10_{10}\right)}$ & \\
\hline frequency & 824.235 & 217.817 & 254.102 & 290.380 & 344.778 & 286.34 & 219.949 & 340.714 & 254.573 & 336.597 & \\
\hline$E_{\text {low }}$ & 247.57 & 39.96 & 55.10 & 72.66 & 103.53 & 1.00 & 16.98 & 45.10 & 60.80 & 88.08 & \\
\hline WX Psc & $<92.2$ & 3.2 & $<1.2$ & 5.4 & 4.8 & $<0.9$ & 1.6 & $<1.4$ & $<2.0$ & {$[0.9]$} & \\
\hline W Aql & [22.3] & - & - & 1.2 & [3.4] & - & $<1.2$ & $<3.4$ & - & - & \\
\hline II Lup & - & - & - & - & 7.8 & - & - & $<4.2$ & - & - & \\
\hline V Cyg & - & 0.9 & $<14.8$ & - & - & - & $<1.3$ & - & - & - & \\
\hline
\end{tabular}

Little interferometric data exist for the molecules that we have observed in any circumstellar envelope. We note, however, that $4^{\prime \prime}-6^{\prime \prime}$ resolution observations of the $\mathrm{HCN} J=1-0$ line in the Mira variables TX Cam and IK Tau (Marvel 2005) barely resolve the emission distibutions in these objects. Since, first, those objects are closer to the Sun than our target stars and, second, our higher exciation lines most likely arise from more compact regions than the 1-0 line, the column densities derived are strict lower limits.

Assuming that the lines are optically thin and that the excitation temperature, $T_{\mathrm{ex}}$, between upper and lower level is such that $T_{\mathrm{ex}} \gg T_{\mathrm{bg}}$ (with $T_{\mathrm{bg}}$ the temperature of any background 

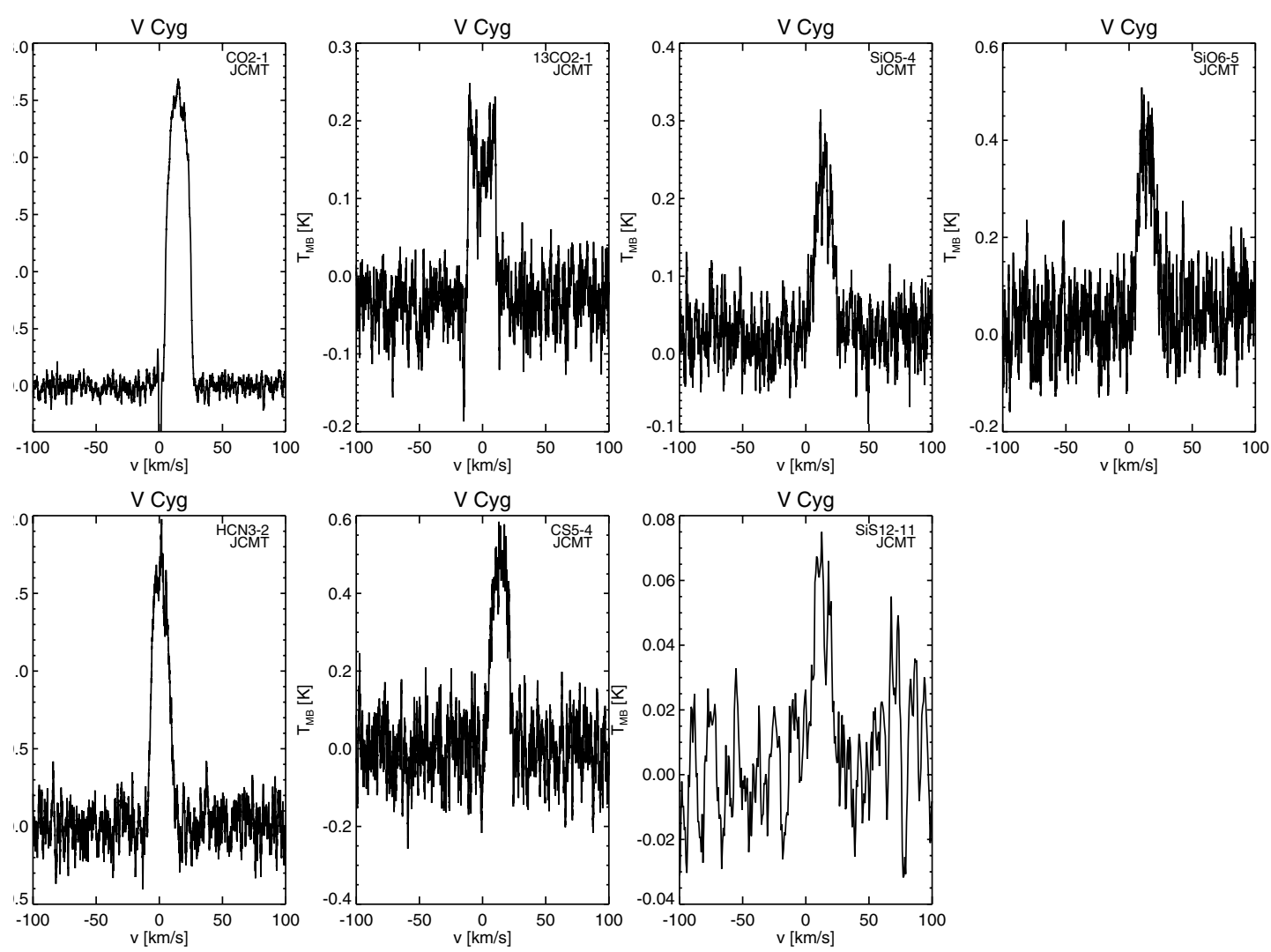

Fig. 4. Molecular emission detected with JCMT in the C-rich AGB V Cyg.

Table 2. Velocity-integrated intensities (in $\mathrm{K} \mathrm{km} \mathrm{s}^{-1}$ ), upper state column densities in $\mathrm{cm}^{-2}$ calculated using Eq. (2) and critical densites in $\mathrm{cm}^{-3}$ for the detected multiple-transitions "parent" molecules used in the excitation analysis. In a few cases, extra integrated-intensity values (listed in italics) as found in literature are added to perform the excitation analysis. Literature references are given in the footnote.

\begin{tabular}{|c|c|c|c|c|c|c|c|c|c|}
\hline & & & & & 2) & 110 & 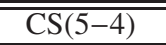 & 7 & 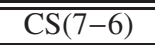 \\
\hline $\begin{array}{cc}\text { WX Psc } & \int T_{\mathrm{mb}} \mathrm{d} \\
N_{\mathrm{u}}\end{array}$ & $\begin{array}{c}9.2 \\
96 \times 10^{12} \\
\end{array}$ & 7.91 & $\begin{array}{r}10 \\
5.15 \times \\
\end{array}$ & & $\begin{array}{c}9.9 \\
96 \times 10^{12}\end{array}$ & $\begin{array}{c}8.9 \\
4.22 \times 10^{12} \\
\end{array}$ & $\begin{array}{c}2.4 \\
53 \times 10^{12} \\
\end{array}$ & $\begin{array}{r}{[0} \\
1.08 \\
\end{array}$ & $\begin{array}{r}1 . \\
41 \times\end{array}$ \\
\hline $\begin{array}{c}\int T_{\mathrm{mb}} \mathrm{d} \\
N_{\mathrm{u}}\end{array}$ & $\begin{array}{c}7.1 \\
19 \times 10^{12} \\
\end{array}$ & $\begin{array}{c}8.9 \\
6.36 \times 10^{12} \\
\end{array}$ & $\begin{array}{c}5.2 \\
2.57 \times 10^{12} \\
\end{array}$ & & $\begin{array}{c}21.7 \\
1.74 \times 10^{13} \\
\end{array}$ & $\begin{array}{c}12.2 \\
5.81 \times 10^{12} \\
\end{array}$ & $\begin{array}{c}8.5^{a} \\
8.95 \times 10^{12} \\
\end{array}$ & $\begin{array}{c}2.3 \\
3.03 \times 10^{12} \\
\end{array}$ & $\begin{array}{c}4.6 \\
5.00 \times 10^{12} \\
\end{array}$ \\
\hline $\begin{array}{c}\int T_{\mathrm{mb}} \mathrm{C} \\
N_{\mathrm{u}} \\
\end{array}$ & & & $\begin{array}{c}19.62 \\
6.96 \times 10^{12} \\
\end{array}$ & $\begin{array}{c}20.4^{b} \\
8.74 \times 10^{12} \\
\end{array}$ & $\begin{array}{c}139.76^{c} \\
1.24 \times 10^{14} \\
\end{array}$ & $\begin{array}{c}60.69 \\
2.88 \times 10^{13} \\
\end{array}$ & $\begin{array}{c}32.34^{c} \\
6.85 \times 10^{13} \\
\end{array}$ & $\begin{array}{r}20 \\
2.61> \\
\end{array}$ & $\begin{array}{c}35.24 \\
3.83 \times 10^{13} \\
\end{array}$ \\
\hline $\begin{array}{c}\int T_{\mathrm{mb}} \\
N_{\mathrm{u}} \\
\end{array}$ & $\begin{array}{c}3.8 \\
3.33 \times 10^{12} \\
\end{array}$ & $\begin{array}{c}5.1 \\
3.51 \times 10^{12} \\
\end{array}$ & & $\begin{array}{c}8.4^{d} \\
5.28 \times 10^{12} \\
\end{array}$ & $\begin{array}{c}25.7 \\
2.06 \times 10^{13} \\
\end{array}$ & $\begin{array}{c}31.9^{d} \\
1.51 \times 10^{13} \\
\end{array}$ & $\begin{array}{c}7.4 \\
1.41 \times 10^{13} \\
\end{array}$ & & \\
\hline $\begin{array}{l}n_{\text {crit }} \\
n_{\text {crit }} \\
n_{\text {crit }}\end{array}$ & $\begin{array}{l}3.06 \times 10^{6} \\
2.00 \times 10^{6} \\
1.22 \times 10^{6}\end{array}$ & $2.10 \times 10^{6}$ & $3.44 \times 10^{6}$ & $5.12 \times 10^{6}$ & $\begin{array}{l}5.83 \times 10^{6} \\
4.22 \times 10^{6} \\
2.35 \times 10^{6}\end{array}$ & $\begin{array}{l}1.32 \times 10^{7} \\
9.63 \times 10^{6} \\
5.65 \times 10^{6}\end{array}$ & $\begin{array}{l}1.85 \times 10^{6} \\
1.35 \times 10^{6} \\
8.35 \times 10^{5}\end{array}$ & $\begin{array}{l}3.22 \times 10^{6} \\
2.37 \times 10^{6} \\
1.48 \times 10^{6}\end{array}$ & $\begin{array}{l}5.09 \times 10^{6} \\
3.76 \times 10^{6} \\
2.37 \times 10^{6}\end{array}$ \\
\hline
\end{tabular}

${ }^{a}$ Bujarrabal et al. (1994); ${ }^{b}$ Schöier et al. (2006a); ${ }^{c}$ Woods et al. (2003); ${ }^{d}$ Bieging et al. (2000).

${ }^{\dagger}$ Line detected however with small $\mathrm{S} / \mathrm{N}$ so that the integrated intensity is quite uncertain.

source, e.g. $2.7 \mathrm{~K}$ ), the integration of the standard radiative transfer equation shows that (Goldsmith \& Langer 1999)

$N_{\mathrm{u}}=\frac{1.67 \times 10^{14}}{\nu \mu^{2}}\left(\int T_{\mathrm{b}} \mathrm{d} v\right)$,

with $N_{\mathrm{u}}$ the column density of the upper transition state in $\mathrm{cm}^{-2}$, $\mu$ the transition dipole moment in Debye, $v$ the transition frequency in $\mathrm{GHz}, T_{\mathrm{mb}}$ the main-beam temperature in Kelvin and $v$ the velocity in $\mathrm{km} \mathrm{s}^{-1}$. For rotational transitions, the transition moment for diatomic and polyatomic molecules is given by

$\mu^{2}(J+1, J)=\frac{J+1}{2 J+3} \mu_{\mathrm{e}}^{2}$, where $\mu_{\mathrm{e}}$ is the permanent dipole moment of the molecule. Using the velocity-integrated intensities of Table 1, we calculate the resulting upper state column densities (see Table 2).

Assuming purely collisional excitation, and ignoring radiation trapping, one can derive by solving the statistical equilibrium equation that (Tielens 2005)

$\frac{N_{1}}{N_{\mathrm{u}}}=\frac{g_{1}}{g_{\mathrm{u}}}\left(\frac{n_{\text {crit }}}{n_{\mathrm{H}_{2}}}+1\right) \exp \left(h v / k T_{\text {kin }}\right)$

where $N_{\mathrm{u}}, N_{\mathrm{l}}, g_{\mathrm{u}}, g_{\mathrm{l}}$ are the upper and lower state column densities and degeneracies, $T_{\text {kin }}$ the kinetic temperature, $n_{\text {crit }}$ the 

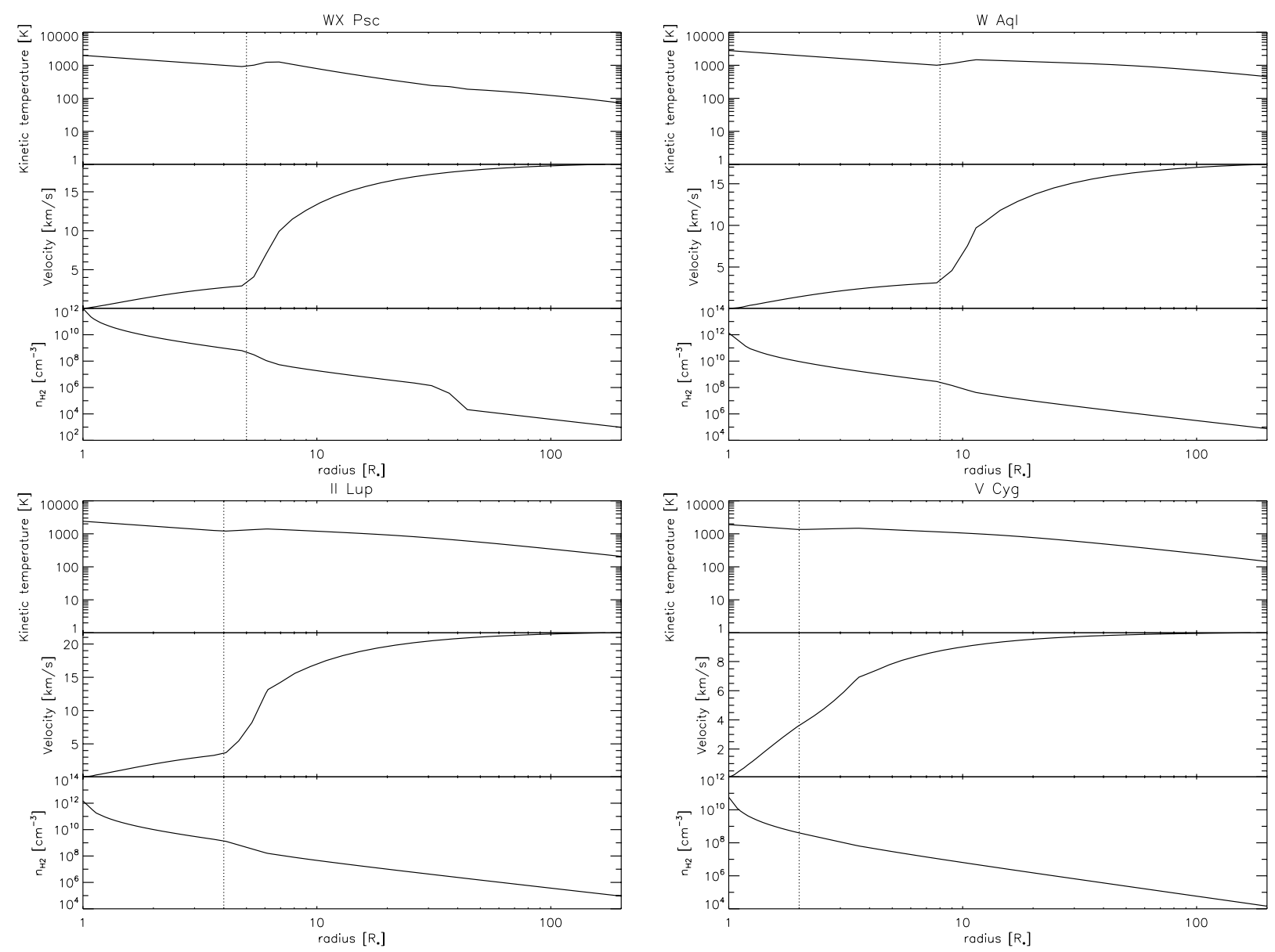

Fig. 5. The structure of the CSE as derived using the GASTRoNOoM-code is shown for the four studied targets WX Psc, W Aql, II Lup, and V Cyg. Since the focus is on the inner and intermediate regions, only the first $200 R_{\star}$ are displayed. Upper panel: estimated temperature profile, middle panel: estimated gas velocity structure, and bottom panel: estimated hydrogen density $n_{\mathrm{H}_{2}}$. The dashed line indicates the dust condensation radius, $R_{\text {inner }}$. The variable mass-loss rate of WX Psc, mostly noticeable in the hydrogen density, is discussed in Decin et al. (2007).

critical density, and $n_{\mathrm{H}_{2}}$ the hydrogen density. For a multi-level system, the critical density is given by (Tielens 2005)

$n_{\text {crit }}=\frac{\sum_{l<u} A_{u l}}{\sum_{l \neq u} \gamma_{u l}}$

where $A_{u l}$ represents the Einstein coefficient, and $\gamma_{u l}$ the collisional rate coefficients. Critical densities were calculated at differing temperatures using the data available in the LAMDA-database (Schöier et al. 2005) (see Table 2). Applying Eq. (4) together with the column densities from Table 2, the minimum density requirements for $n_{\mathrm{H}_{2}}$ to achieve the observed number-density ratios are tabulated in Table 3 . Note that the listed values are at $T_{\text {kin }}=300 \mathrm{~K}$, and that for lower temperatures the critical density increases.

The gas temperature, density and velocity structure was calculated in a self-consistent way using the GASTRoNOoM-code (Decin et al. 2006) to determine where in the envelope the gas density falls below the density requirements given in Table 3 (see Fig. 5). The assumed stellar parameters are listed in Table 4, and the derived (maximum) radius of the emitting region is listed in Table 3. If collisional excitation is assumed, it appears from Table 3 that the "parent" molecules SiO, HCN and CS are excited in the inner $\left(\$ 20 R_{*}\right)$ and intermediate $\left(\$ 70 R_{*}\right)$ regions of the circumstellar envelope and trace regions after the dust condensation zone where they have been injected from deeper layers.
Table 3. For each target, the first row lists the minimum number density for $n_{\mathrm{H}_{2}}$ in $\mathrm{cm}^{-3}$ as derived using Eq. (4) at $T_{\text {kin }}=300 \mathrm{~K}$ and the second row gives the corresponding maximum radius for the emission regions obtained using the GASTRoNOoM-code. The third row gives the maximum radius for the emitting region calculated using Eq. (6).

\begin{tabular}{llccc}
\hline \hline & & $\mathrm{SiO}$ & $\mathrm{HCN}$ & $\mathrm{CS}$ \\
\hline \multirow{4}{*}{ WX Psc } & $n_{\mathrm{H}_{2}}$ & $5.0 \times 10^{6}$ & $4.4 \times 10^{6}$ & $9.0 \times 10^{5}$ \\
& $R$ (Eq. (4)) & $17 R_{*}$ & $19 R_{*}$ & $33 R_{*}$ \\
& $R$ (Eq. (6)) & $4.5 R_{*}$ & $6 R_{*}$ & $7.5 R_{*}$ \\
\hline \multirow{3}{*}{ W Aql } & $n_{\mathrm{H}_{2}}$ & $2.1 \times 10^{6}$ & $2.1 \times 10^{6}$ & $6.34 \times 10^{5}$ \\
& $R$ (Eq. (4)) & $40 R_{*}$ & $40 R_{*}$ & $70 R_{*}$ \\
& $R$ (Eq. (6)) & $9 R_{*}$ & $10 R_{*}$ & $11 R_{*}$ \\
\hline \multirow{4}{*}{ II Lup } & $n_{\mathrm{H}_{2}}$ & $2.7 \times 10^{7}$ & $1.3 \times 10^{6}$ & $7.6 \times 10^{5}$ \\
& $R$ (Eq. (4)) & $13 R_{*}$ & $53 R_{*}$ & $70 R_{*}$ \\
& $R$ (Eq. (6)) & $2.5 R_{*}$ & $13 R_{*}$ & $10 R_{*}$ \\
\hline \multirow{3}{*}{ V Cyg } & $n_{\mathrm{H}_{2}}$ & $2.8 \times 10^{7}$ & $8.7 \times 10^{6}$ & \\
& $R$ (Eq. (4)) & $5 R_{*}$ & $9 R_{*}$ & \\
& $R$ (Eq. (6)) & & $4 R_{*}$ & \\
\hline
\end{tabular}

It is also of interest to consider the case where collisional excitation is ignored, and the molecules are excited by infrared radiation from the star. One can derive that (Tielens 2005)

$\frac{N_{\mathrm{l}}}{N_{\mathrm{u}}}=\frac{g_{1}}{g_{\mathrm{u}}} \frac{\exp \left(h v / k T_{*}\right)-1}{W}+1$, 
Table 4. Stellar parameters used as input for the GASTRoNOoM-code. The terminal velocity, $v_{\infty}$, is derived from the CO lines; the stellar radius from the stellar luminosity and temperature. The envelope density decreases as $\sim r^{-2}$. Literature references are given in the footnote.

\begin{tabular}{lcccc}
\hline \hline & WX Psc & W Aql & II Lup & V Cyg \\
\hline$T_{*}[\mathrm{~K}]$ & $2000^{a}$ & 2800 & $2400^{f}$ & $1900^{f}$ \\
$R_{*}\left[10^{13} \mathrm{~cm}\right]$ & 5.5 & 2.4 & 3.8 & 5.1 \\
$L_{*}\left[10^{3} L_{\odot}\right]$ & $10^{a}$ & $6.8^{b}$ & $8.8^{f}$ & $6.3^{f}$ \\
{$[\mathrm{CO}] /\left[\mathrm{H}_{2}\right]\left[10^{-4}\right]$} & $3^{c}$ & $6^{c}$ & $8^{c}$ & $8^{c}$ \\
distance $[\mathrm{pc}]$ & $833^{a}$ & $230^{d}$ & $500^{f}$ & $310^{f}$ \\
$R_{\text {inner }}\left[R_{*}\right]$ & $5^{a}$ & $8^{e}$ & $4^{f}$ & $2^{f}$ \\
$v_{\infty}\left[\mathrm{km} \mathrm{s}^{-1}\right]$ & 18 & 17.5 & 21 & 10.5 \\
$\dot{M}\left[10^{-6} M_{\odot} \mathrm{yr}^{-1}\right]$ & $6^{a, \dagger}$ & $2.5^{b}$ & $9^{f}$ & $1.2^{g}$ \\
\hline
\end{tabular}

${ }^{a}$ Decin et al. (2007); ${ }^{b}$ Ramstedt et al. (2006); ${ }^{c}$ Knapp \& Morris (1985); ${ }^{d}$ Bieging et al. (2000); ${ }^{e}$ Danchi et al. (1994); ${ }^{f}$ Schöier et al. (2006a);

${ }^{g}$ Schöier \& Olofsson (2001).

${ }^{\dagger}$ Refers to the inner region (A) in Decin et al. (2007).

where $W$ is the geometrical dilution, being $\left(R_{*} / 2 R\right)^{2}$ when $R \gg$ $R_{*}$. As seen from Table 3, radiative excitation constrains the emitting zone for $\mathrm{SiO}$ to $\lesssim 9 R_{*}$, for $\mathrm{HCN} \lesssim 13 R_{*}$, and for CS $\lesssim$ $11 R_{*}$. In general, these regions are smaller than derived in the case of collisional excitation, indicating that the radiation field of the star does not have enough energy to sustain the excitation. An analogous expression as for Eq. (6) can be derived for a radiation field characterized by a dust temperature $T_{\mathrm{d}}$ at the condensation radius $R_{\text {inner }}$, with the dilution factor then being $\left(R_{\text {inner }} / 2 R\right)^{2}$. Using the dust condensation radii listed in Table 4 and a dust temperature of $800 \mathrm{~K}$ the derived emitting regions are a factor 3.2 larger for WX Psc, a factor 4.3 for W Aql, a factor 4 for II Lup, and a factor 2 for V Cyg, resulting in similar radii as in the case of collisional excitation.

Although the numbers in Table 3 can only be used as rough guidelines, they suggest in both cases a sequence in the excitation pattern, $\mathrm{SiO}$ being the species emitted the closest to the star, followed by $\mathrm{HCN}$ and $\mathrm{CS}$.

\section{Conclusions}

From the above analysis, one can draw the following conclusions:

1. The observations reported in this letter confirm the status of "parent" molecules for $\mathrm{CO}, \mathrm{SiO}, \mathrm{HCN}$, and CS in AGB stars, whose observed molecular lines form close to the star in support of the theoretical predictions of Cherchneff (2006). The excitation analysis suggests that $\mathrm{SiO}$ is emitted closest to the star, followed by HCN and CS.

2. High-excitation lines of SiS are detected in all stars, implying that $\mathrm{SiS}$ too forms close to the star, whatever the stage of stellar evolution. However, a thorough line analysis is necessary to prove or disprove that $\mathrm{SiS}$ is more abundant in $\mathrm{C}$ stars than in O-rich Miras, as predicted by Cherchneff (2006).

3. SO appears to be typical of O-rich AGBs only. With only two excitation lines detected, no conclusion can be drawn on the locus of SO formation. However, the $\mathrm{SO}\left(10_{11}-10_{10}\right)$ line has an estimated velocity of only $10 \mathrm{~km} \mathrm{~s}^{-1}$ in WX Psc when the wind terminal velocity for this object is $19.2 \mathrm{~km} \mathrm{~s}^{-1}$. This fact suggests that the line excitation occurs close to the star. The inner chemistry of O-rich AGB envelopes appears to be as rich, if not richer, than that of $\mathrm{C}$-rich stars.

A detailed line analysis of the present data coupled to further observational campaigns with JCMT and APEX are planned to corroborate these results.

Acknowledgements. L.D. and S.D. acknowledge financial support from the Fund for Scientific Research - Flanders (Belgium), I.C. acknowledges support from the Swiss National Funds for Science through a Marie-Heim-Vögtlin Fellowship, and S.H. acknowledges financial support from the Interuniversity Attraction Pole of the Belgian Federal Science Policy P5/36. We thank Remo Tilanus (JCMT) for his support during the observations and reduction of the data.

\section{References}

Bieging, J. H., Shaked, S., \& Gensheimer, P. D. 2000, ApJ, 543, 897 Bujarrabal, V., Fuente, A., \& Omont, A. 1994, A\&A, 285, 247 Cherchneff, I. 2006, A\&A, 456, 1001

Danchi, W. C., Bester, M., Degiacomi, C. G., Greenhill, L. J., \& Townes, C. H. 1994, AJ, 107, 1469

Decin, L., Hony, S., de Koter, A., et al. 2006, A\&A, 456, 549

Decin, L., Hony, S., de Koter, A., et al. 2007, A\&A, 475, 233

Duari, D., Cherchneff, I., \& Willacy, K. 1999, A\&A, 341, L47

Goldsmith, P. F. \& Langer, W. D. 1999, ApJ, 517, 209

Güsten, R., Nyman, L. Å., Schilke, P., et al. 2006, A\&A, 454, L13

Justtanont, K., de Jong, T., Helmich, F. P., et al. 1996, A\&A, 315, L217

Knapp, G. R., \& Morris, M. 1985, ApJ, 292, 640

Marvel, K. B. 2005, AJ, 130, 261

Morris, M. 1975, ApJ, 197, 603

Olofsson, H., Eriksson, K., Gustafsson, B., \& Carlstrom, U. 1993, ApJS, 87, 267

Ramstedt, S., Schöier, F. L., Olofsson, H., \& Lundgren, A. A. 2006, A\&A, 454, L103

Ryde, N., Eriksson, K., Gustafsson, B., Lindqvist, M., \& Olofsson, H. 1998, Ap\&SS, 255, 301

Schöier, F. L., \& Olofsson, H. 2001, A\&A, 368, 969

Schöier, F. L., van der Tak, F. F. S., van Dishoeck, E. F., \& Black, J. H. 2005, A\&A, 432, 369

Schöier, F. L., Fong, D., Olofsson, H., Zhang, Q., \& Patel, N. 2006a, ApJ, 649, 965

Schöier, F. L., Olofsson, H., \& Lundgren, A. A. 2006b, A\&A, 454, 247

Schöier, F. L., Bast, J., Olofsson, H., \& Lindqvist, M. 2007, A\&A, 473, 871

Tielens, A. G. G. M. 2005, The Physics and Chemistry of the Interstellar Medium (Cambridge University Press)

Tsuji, T. 1973, A\&A, 23, 411

Willacy, K., \& Cherchneff, I. 1998, A\&A, 330, 676

Woods, P. M., Schöier, F. L., Nyman, L.-Å., \& Olofsson, H. 2003, A\&A, 402, 617 